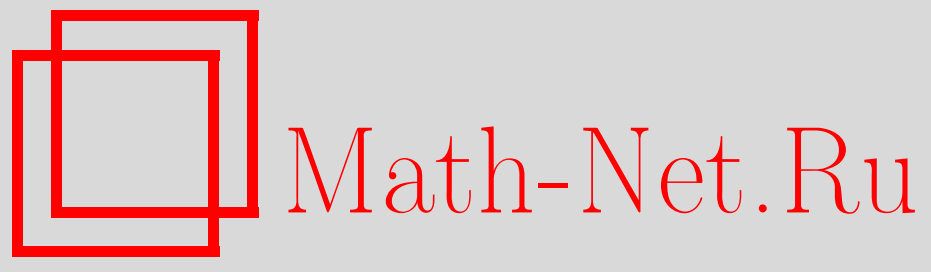

С. И. Адян, А. Л. Семёнов, В. А. Успенский, Андрей Альбертович Мучник (некролог), УМН, 2007, том 62, выпуск 4, 140-144

DOI: https://doi.org/10.4213/rm7922

Использование Общероссийского математического портала Math-Net.Ru подразумевает, что вы прочитали и согласны с пользовательским соглашением http://www.mathnet.ru/rus/agreement

Параметры загрузки:

IP : 3.80 .253 .173

26 апреля 2023 г., $14: 25: 54$

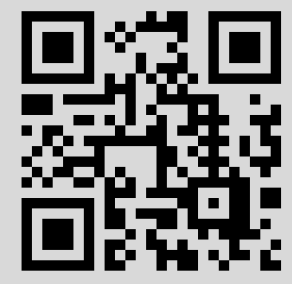




\section{Андрей Альбертович Мучник}

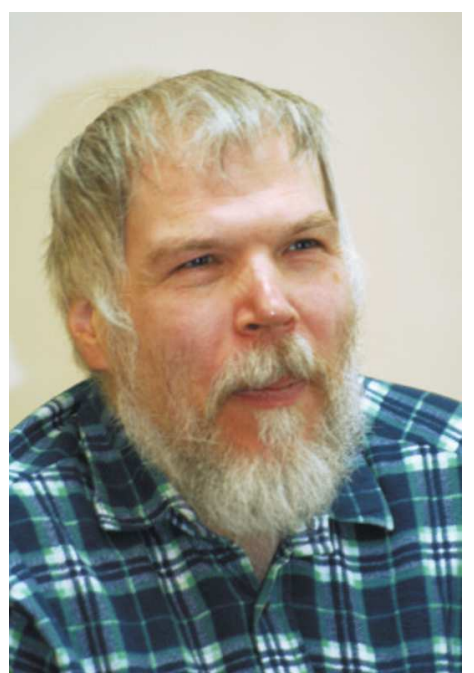

Андрей Альбертович Мучник родился в семье математиков. Его родители Надежда Митрофановна Ермолаева и Альберт Абрамович Мучник были учениками Петра Сергеевича Новикова. Альберт Абрамович Мучник в 1956 г., будучи аспирантом П. С. Новикова, решил знаменитую проблему Поста - о существовании нетривиальной перечислимой степени сводимости по Тьюрингу. Первая работа Андрея Мучника относилась к дифференциальным уравнениям и была выполнена на втором курсе, когда он был участником семинара Е.М. Ландиса и Ю.С. Ильяшенко. На его выбор математической логики и теории алгоритмов в качестве основной области исследований оказала влияние склонность Андрея к логическому, философскому осмыслению материального и идеального мира; его научным руководителем стал ученик Ал. А. Мучника - А. Л. Семёнов.

В своей дипломной работе [1], защищенной на кафедре математической логики МГУ в мае 1981 г., Ан. А. Мучник решил проблему устранения трансфинитной индукции в доказательстве важнейшей теоремы Рабина о разрешимости монадической теории нескольких следований. Эта проблема была поставлена М. Рабином на Международном математическом конгрессе в Ницце в 1970 г. (см. [16]). Доказательство Мучника рассказывалось на спецкурсах кафедры математической логики мехмата МГУ А.Л. Семёновым в 1979/80 учебном году и А. Г. Драгалиным в 1980/81 году. Сходное решение проблемы Рабина получили Ю. Гуревич и Л. Харрингтон (предварительная, краткая версия их работы опубликована в [17]). Тогда же Ан. А. Мучник использовал свой метод для доказательства обобщения теоремы Шелаха-Ступа, в свою очередь обобщающей теорему Рабина. Этот результат Мучника (без доказательства) опубликован в [18]. В теореме Мучника, в дальнейшем использованной рядом авторов, дерево строится не над конечным множеством или натуральным рядом, как у Рабина, а над произвольной структурой с разрешимой монадической теорией, кроме того, допускается отношение равенства между двумя соседними элементами пути на дереве (см. [19]).

В области разрешимых логических теорий Ан. Мучнику принадлежит также замечательный результат, связанный с известной теоремой Кобхэма-Семёнова. В работе [20] А. Кобхэм доказал, что всякое множество чисел, автоматное в двух системах счисления с мультипликативно-независимыми основаниями, является объединением конечного числа арифметических прогрессий. А.Л. Семёнов в работе [21] обобщил этот результат со случая множеств на случай многоместных отношений. Ан. Мучник получил указанное утверждение как непосредственное следствие из доказанной им 
теоремы о самовыразимости арифметики сложения (см. [5]). Эта теорема Мучника в дальнейшем использовалась многими авторами. Отметим, что ее доказательство существенно короче, чем исходное доказательство теоремы Семёнова.

Ряд результатов Мучника, начиная с красивого доказательства теоремы Шутценберже (см. [2]), относится к теории формальных грамматик и языков. В частности, он получил интересные алгоритмические результаты, относящиеся к контекстносвободным грамматикам (см. [4]), развивая изложенный в статье А. Л. Семёнова [22] подход, основная идея которого также принадлежала Ал. А. Мучнику.

В работе [3] Ан. Мучник доказал общую теорему, устанавливающую, что класс релятивизуемых (т. е. не зависящих от использованного оракула) утверждений качественной теории алгоритмов может быть охарактеризован с помощью некоторого естественного класса игр. В дальнейшем ряд доказательств Ан. Мучника состоял именно в построении стратегии в соответствующей игре [6], [9], [15].

Последнее десятилетие основные научные интересы Ан. Мучника относились к теории сложности объектов (колмогоровской сложности). Многие его результаты в этой теории уже стали классическими. К ним относится, в частности, изучение различных вариантов понятия случайности. Мучник дал наиболее общее на сегодняшний день естественное определение непредсказуемости для бесконечных последовательностей. А именно, последовательность называется непредсказуемой, если не существует алгоритма, который, начав с единичного капитала, читая члены последовательности в любом порядке и делая ставки в пределах текущего капитала на значения непрочитанных членов, выигрывает в пределе бесконечно много (см. [7]).

Колмогоров начал свои исследования в данной области с фундаментального вопроса о применимости законов теории вероятностей к явлениям реального мира. Почему, например, последовательность, полученная в результате большого числа бросаний симметричной монеты, с хорошей точностью удовлетворяет закону больших чисел (т. е. частота выпадений единицы мало отклоняется от $1 / 2)$ ? Колмогоров предложил следующий подход к этому явлению: постулируем, что последовательность, полученная достаточным количеством бросаний, имеет большую колмогоровскую сложность. Отсюда можно вывести, что она с хорошей точностью удовлетворяет закону больших чисел. То же самое верно для любой ее достаточно длинной подпоследовательности, отобранной алгоритмом малой колмогоровской сложности (отбирающему алгоритму разрешается читать члены последовательности в любом порядке и принимать решение об отборе непрочитанных членов на основе значений прочитанных членов). Вопрос о точном соотношении сложности исходной последовательности, сложности отбирающего алгоритма и степени выполнения для отобранной подпоследовательности закона больших чисел был поставлен Колмогоровым в его работе [23], начавшей исследования, теперь относящиеся к "колмогоровской теории сложности". Через 20 лет в предисловии к русскому переводу этой работы Колмогоров подчеркнул важность решения этого вопроса. Еще через 20 лет Ан. Мучник заинтересовался проблемой Колмогорова, довел до логического завершения построения исходной статьи и предложил путь, на котором проблема Колмогорова была решена (см. [11]-[13]). Указанная статья Колмогорова относилась к "комбинаторному" подходу к сложности (и случайности). В ней же он, в нескольких фразах, наметил и получивший дальнейшее развитие "алгоритмический" подход. Как оказалось, для "комбинаторных" теорем Колмогорова существуют "алгоритмические” аналоги (см. [12]). Они в определенном смысле завершают программу, сформулированную Колмогоровым в основополагающей статье. Внимание к первоисточникам, попытка понять мотивы, побудившие предшественников к тем или иным рассмотрениям, стремление пройти их путь характерны для всей математической деятельности Ан. Мучника.

Ранее Ан. Мучник получил аналогичные результаты для бесконечных последовательностей. А именно, он доказал, что если сложность начала длины $n$ данной бес- 
конечной двоичной последовательности растет не быстрее, чем $c n$ (где $c$ - константа, строго меньшая единицы), то существует алгоритм, отбирающий из нее бесконечную подпоследовательность, не удовлетворяющую закону больших чисел (см. [7]). Тем самым было показано, что закон больших чисел достаточно точно характеризует случайность по Колмогорову, и была опровергнута гипотеза Колмогорова о том, что существует стохастическая последовательность, у которой энтропия начальных отрезков растет логарифмически (см. [24]).

Вот пример результата, который можно отнести к области "алгебры и логики сложности": пусть даны два произвольных слова $A$ и $B$, тогда существует программа, преобразующая $B$ в $A$, с длиной, близкой к минимальной (среди всех программ, преобразующих $B$ в $A$ ), имеющая малую сложность относительно $A$ (см. [9]). В работах [10], [14] устанавливается связь между колмогоровской сложностью, вычислимым анализом и классической проблематикой Э. Поста.

Выдающиеся способности, результаты и вкус Андрея как математика сочетались с его замечательными качествами как коллеги и партнера по математическому исследованию. Он легко делился своими наблюдениями и результатами, входил в российские и международные авторские коллективы многочисленных публикаций. Его замечания, комментарии и советы содействовали улучшению или послужили отправной точкой для многих работ, обсуждавшихся на Колмогоровском семинаре в МГУ, одним из фактических руководителей которого он являлся с самого начала. На семинаре по алгоритмическим проблемам алгебры С. И. Адяна он часто удивлял участников своим тонким пониманием и интуицией во многих областях, не относящихся к постоянному кругу его интересов. Сотрудничество с Ан. Мучником было принципиальным для многих российских и зарубежных коллег. Его результаты, соображения, идеи рассыпаны по десяткам статей и книг других авторов.

Глубокое понимание математической сути вещей сочеталось у Ан. Мучника с ощущением и привлечением широкого общематематического, общефилософского и просто житейского контекста (к сожалению, он слишком болезненно воспринимал отсутствие логики в окружающем мире). Он обладал способностью объяснять самую разную математику на любом уровне простоты или сложности с одинаковым увлечением. Делал это он с огромной благожелательностью и тактом и, казалось, располагал неограниченным запасом времени...

Ан. Мучник был очень требователен к своим рассуждениям и, особенно, к математическим текстам. Часто это задерживало публикацию на годы, многие из его результатов были записаны и подготовлены к печати его коллегами, к которым он всегда испытывал благодарность за помощь (иногда такая подготовка занимала несколько лет). Некоторые из его работ еще не доведены до публикации, надеемся, что они не будут утеряны. Нормальные условия для математической работы и жизни Андрея Мучника были обеспечены благодаря Российской академии наук (Научному совету по комплексной проблеме "Кибернетика" и ВЦ им. А. А. Дородницына, где он работал) и Институту новых технологий. Его работа была поддержана рядом российских и международных грантов. Из людей, не входивших непосредственно в круг его научного общения, он всегда был благодарен за поддержку А. А. Болибруху.

Андрей Мучник ушел от нас в расцвете творческой активности. В 2006 г. ему была присуждена Премия имени А.Н. Колмогорова Российской академии наук (совместно с А.Л. Семёновым). Но работал он не ради наград и званий, а ради Математики, а еще больше - ради общения, ради интереса и доброго слова окружающих людей. 


\section{Цитируемые работы Ан. А. Мучника}

[1] Игры на бесконечных деревълх и автоматы с тупиками. Новое доказательство разрешимости монадической теории двух следований, Дипломная работа, Кафедра математической логики, Мех.-мат. ф-т МГУ, 1981; Семиотика и информатика, 24, ВИНИТИ, М., 1985, 16-40; англ. пер.: "Games on infinite trees and automata with dead-ends. A new proof for the decidability of the monadic second order theory of two successors", Bull. Eur. Assoc. Theor. Comput. Sci. EATCS, 48 (1992), 220-267.

[2] "О теореме Шютценберже, касающейся моноидов без нетривиальных подгрупп", Математическая логика, математическая лингвистика и теория алгоритмов, Изд-во Калининского гос. ун-та, Калинин, 1983, 65-68.

[3] "Об основных структурах дескриптивной теории алгоритмов", Докл. АН CCCP, 285:2 (1985), 280-283; англ. пер.: "On the basic structures of the descriptive theory of algorithms", Soviet Math. Dokl., 32 (1985), 671-674.

[4] Одно применение действительнозначной интерпретации грамматических рядов, Ин-т новых технологий, М., 1991; англ. пер.: "One application of real-valued interpretation of formal power series", Theoret. Comput. Sci., 290:3 (2003), 1931-1946.

[5] Выразимый критерий выразимости в арифметике Пресбургера и его применения, Ин-т новых технологий, М., 1991; англ. пер.: "The definable criterion for definability in Presburger arithmetic and its applications", Theoret. Comput. Sci., 290:3 (2003), 1433-1444.

[6] "On common information", Theoret. Comput. Sci., 207:2 (1998), 319-328.

[7] "Mathematical metaphysics of randomness", Theoret. Comput. Sci., 207:2 (1998), 263-317 (with A. L. Semenov, V. A. Uspensky).

[8] "Conditional complexity and codes", Theoret. Comput. Sci., 271:1-2 (2002), 97-109.

[9] "Upper semi-lattice of binary strings with the relation " $x$ is simple conditional to $y$ ", Theoret. Comput. Sci., 271:1-2 (2002), 69-95 (with A. Chernov, A. Romashchenko, A. Shen, N. Vereshchagin).

[10] "Kolmogorov entropy in the context of computability theory", Theoret. Comput. Sci., 271:1-2 (2002), 15-35 (with S. Ye. Positselsky).

[11] "Об уточнении оценок Колмогорова, относящихся к датчикам случайных чисел и сложностному определению случайности”, Докл. РАН, 391:6 (2003), 738-740 (совм. с А. Л. Семёновым); англ. пер.: "An improvement of Kolmogorov's estimates related to random number generators and a definition of randomness in terms of complexity", Dokl. Math., 68:1 (2003), 132-134 (with A. L. Semenov).

[12] "О роли закона больших чисел в теории случайности", Проблемы передачи информации, 39:1 (2003), 134-165 (совм. с А.Л. Семёновым); англ. пер.: "On the role of the law of large numbers in the theory of randomness", Probl. Inf. Transm., 39:1 (2003), 119-147 (with A. L. Semenov).

[13] "40 Years of the origin of Kolmogorov randomness theory", Колмогоров и современная математика. Тезисы докладов Междунар. конф., посв. 100-летию со дня рожд. А.Н. Колмогорова (25.04.1903-20.10.1987) (Москва, 2003), Изд-во мех.-матем. ф-та МГУ, М., 2003, 677-678 (with A. L. Semenov).

[14] "Effective bounds for convergence, descriptive complexity, and natural examples of simple and hypersimple sets", Ann. Pure Appl. Logic, 141:3 (2006), 437-441 (with A. Semenov). 
[15] "Non-reducible descriptions for conditional Kolmogorov complexity", Theory and applications of models of computation (Beijing, China, 2006), Lecture Notes in Comput. Sci., 3959, Springer, Berlin, 2006, 308-317 (with A. Shen, M. Ustinov, N. Vereshchagin, M. Vyugin).

\section{Цитируемые работы других авторов}

[16] M. O. Rabin, "Decidability and definability in second-order theories", Actes du Congrès international des mathématiciens (Nice, France, 1970), Vol. 1, Gauthier-Villars, Paris, 1971, 239-244.

[17] Y. Gurevich, L. Harrington, "Trees, automata, and games", Proceedings of the fourteenth annual ACM symposium on theory of computing (San Francisco, USA, 1982), ACM Press, New York, 1982, 60-65.

[18] A. L. Semenov, "Decidability of monadic theories", Mathematical foundations of computer science (Prague, 1984), Lecture Notes in Comput. Sci., 176, Springer, Berlin, 1984, 162-175.

[19] A. Blumensath, S. Kreutzer, "An extension of Muchnik's theorem", J. Logic Comput., 15:1 (2005), 59-74.

[20] A. Cobham, "On the base-dependence of set of numbers recognizable by finite automata", Math. Systems Theory, 3:2 (1969), 186-192.

[21] А. Л. Семёнов, "Пресбургеровость предикатов, регулярных в двух системах счисления", Сиб. матем. журн., 18:2 (1977), 403-418; англ. пер.: А. L. Semenov, "Presburgerness of predicates regular in two number systems", Siberian Math. J., 18:2 (1977), 289-300.

[22] А.Л. Семёнов, "Алгоритмические проблемы для степенных рядов и контекстно-свободных грамматик", Докл. АН ССCP, 212 (1973), 50-52; англ. пер.: A. L. Semenov, "Algorithmic problems for power series and context-free grammars", Soviet Math. Dokl., 14 (1973), 1319-1322.

[23] A. N. Kolmogorov, "On tables of random numbers", Sankhyā Ser. A, 25 (1963), 369-376; Theoret. Comput. Sci., 207:2 (1998), 387-395; pyc. пер.: А. Н. Колмогоров, "О таблицах случайных чисел", Семиотика и информатика, 18, ВИНИТИ, M., 1982, 3-13.

[24] А.Н. Колмогоров, "К логическим основам теории информации и теории вероятностей", Проблемы передачи информачии, 5:3 (1969), 3-7; англ. пер.: A. N. Kolmogorov, "Logical basis for information theory and probability theory", IEEE Trans. Inform. Theory, 14:5 (1968), 662-664. 